\title{
Appendix A: Solutions to Selected Problems
}

Problem 1.1. A given biased RW is defined by $\left\langle\mathbf{s}_{n}\right\rangle=\mathbf{a}$ and $\left\langle s_{n}^{2}\right\rangle=\lambda^{2}$, with $\mathbf{a}$ and $\lambda$ constants. Determine the two rms loci of this biased random walker after $N$ steps in 1D (as in Fig. 1.1) and generalize to 2D and 3D, as function of $a_{x} / \lambda, a_{y} / \lambda$ and in 3D, $a_{z} / \lambda$.

\section{Solution:}

$$
\begin{array}{r}
\left\langle s_{n}\right\rangle=a \quad \text { and } \quad\left\langle s^{2}\right\rangle=\lambda^{2}, \\
\left\langle\left(s_{n}-a\right)^{2}\right\rangle=\left\langle s_{n}^{2}\right\rangle-2\left\langle s_{n}\right\rangle a+a^{2}=\lambda^{2}-a^{2}, \\
\left\langle\sum_{n=1}^{N}\left(s_{n}-a\right)\right\rangle=\left\langle\sum_{n=1}^{N} s_{n}\right\rangle-N a=0 .
\end{array}
$$

The systematic walking distance after $N$ steps is $N a$, so it must be separated out before calculating the rms (root-mean-square) deviation from this "ballistic trajectory":

$$
\begin{aligned}
\left\langle\left(r_{N}-N a\right)^{2}\right\rangle & =\left\langle\left(\sum_{n=1}^{N}\left(s_{n}-a\right)\right)^{2}\right\rangle \\
& =N\left(\lambda^{2}-a^{2}\right)+2 \sum_{n=1}^{N-1} \sum_{m=n+1}^{N}\left\langle\left(s_{n}-a\right)\left(s_{m}-a\right)\right\rangle=N\left(\lambda^{2}-a^{2}\right)
\end{aligned}
$$

since for $n \neq m\left\langle\left(s_{n}-a\right)\left(s_{m}-a\right)\right\rangle=\left\langle\left(s_{n}-a\right)\right\rangle\left\langle\left(s_{m}-a\right)\right\rangle=0$.

In 1D:

$$
r_{r m s}=N a \pm \sqrt{N} \sqrt{\lambda^{2}-a^{2}}
$$


In 2D: all points lie within a circle centered at $N \vec{a}$, of radius $r_{r m s}=$ $\sqrt{N} \sqrt{\lambda^{2}-a^{2}}$.

In 3D: all points lie within a sphere centered at $N \vec{a}$, of radius $r_{r m s}=$ $\sqrt{N} \sqrt{\lambda^{2}-a^{2}}$

Problem 1.2. Find a formula for area of unit sphere $A(d)$ in $d$ dimensions.

$\left[\right.$ Hint : using $\mathbf{r}^{2}=r_{1}^{2}+r_{2}^{2}+\cdots+r_{d}^{2}$, compute :

$$
\begin{aligned}
\int d^{d} r e^{-\mathbf{r}^{2}} & =A(d) \int_{0}^{\infty} d r r^{d-1} e^{-r^{2}}=\frac{A(d)}{2} \int_{0}^{\infty} d t t^{\left(\frac{d}{2}-1\right)} e^{-t} \\
& \left.=\left\{\int_{-\infty}^{\infty} d x e^{-x^{2}}\right\}^{d} \cdot\right]
\end{aligned}
$$

Evaluate $A(d)$ explicitly for $d=1,2,3,4,5$.

\section{Solution:}

$$
\begin{aligned}
& A_{d}=2 \frac{\left\{\int_{-\infty}^{\infty} d x \cdot e^{-x^{2}}\right\}^{d}}{\int_{0}^{\infty} d t \cdot t^{\left(\frac{d}{2}-1\right)} e^{-t}}=\frac{2 \pi^{\frac{d}{2}}}{\Gamma\left(\frac{d}{2}\right)} \\
& \int \frac{2 \pi^{\frac{d}{2}}}{\left(\frac{d}{2}-1\right) !} \quad \text { if } d \text { is even } \\
& = \begin{cases}\left(\frac{d}{2}-1\right) ! & \text { if } d=1 \\
2 & \text { if } d \text { odd and } \geq 3 \\
\text { or } \pi^{\frac{d-1}{2}} \frac{2^{d-1}\left(\frac{d-3}{2}\right) !}{(d-2) !} & \end{cases} \\
& A_{1}=2, \quad A_{4}=2 \pi^{2} \\
& A_{2}=2 \pi, \quad A_{5}=8 \pi^{2} / 3 \\
& A_{3}=4 \pi \text {, }
\end{aligned}
$$


Problem 1.3. Estimate the fractional errors in Stirling's approximation arising from the two sources: the neglect of the next term $\left(1 / 3 ! g^{\prime \prime \prime}\left(t-t_{0}\right)^{3}\right)$ in the expansion of $g(t)$ and the approximation of $-N$ by $-\infty$ in the limits of integration. Compare Stirling's result with the exact values of $\Gamma(z)$ for $z=3,3.5,10$ and 10.5 and obtain the fractional errors numerically. How well do they agree with your estimate?

\section{Solution:}

a) $\exp [g(t)]=t^{N} e^{-t}=\exp [N \log t-t]$

$$
\begin{gathered}
g^{\prime}(t)=-1+\frac{N}{t}=0 \text { at } t=t_{0} . \text { Also, } g^{\prime \prime}(t)=-\frac{N}{t^{2}} ; g^{\prime \prime \prime}(t)=\frac{2 N}{t^{3}}, \text { etc. } \\
\therefore t_{0}=N ; g^{\prime}=0 ; g^{\prime \prime}\left(t_{0}\right)=-\frac{N}{N^{2}}=-\frac{1}{N} ; g^{\prime \prime \prime}\left(t_{0}\right)=\frac{2}{N^{2}}, \text { etc. } \\
\Gamma(N+1)=N !=\int_{0}^{\infty} t^{N} e^{-t} d t=\int_{0}^{\infty} \exp [g(t)] d t .
\end{gathered}
$$

Plug in $g$ and expressions for $g$ and $t_{0}$ in terms of $N$ :

$$
\begin{aligned}
& g(t)=g\left(t_{0}\right)+\left[\frac{\left(t-t_{0}\right)^{2}}{2 !} g^{\prime \prime}\left(t_{0}\right)\right]+\left[\frac{\left(t-t_{0}\right)^{3}}{3 !} g^{\prime \prime \prime}\left(t_{0}\right)\right]+\cdots \\
& g(t)=g\left(t_{0}\right)-\left[\frac{(t-N)^{2}}{2 N}\right]+\left[\frac{(t-N)^{3}}{3 N^{2}}\right]+\cdots
\end{aligned}
$$

where $g\left(t_{0}\right)=N \log N-N$.

$$
N !=\int_{0}^{\infty} \exp [g(t)] d t=\int_{0}^{\infty} \exp \left[N \log N-N-\frac{(t-N)^{2}}{2 N}+\frac{(t-N)^{3}}{3 N^{2}}\right] d t
$$

taking 3 first terms

$$
=\exp [N \log N-N] \int_{0}^{\infty} \exp \left[-\frac{(t-N)^{2}}{2 N}+\frac{(t-N)^{3}}{3 N^{2}}\right] d t
$$

then shift range of integration by $N$ and expand the small term in $t^{3} / N^{2}$ :

$$
=\exp [N \log N-N] \int_{-N}^{\infty}\left[1+\frac{t^{3}}{3 N^{2}}+\cdots\right] \exp \left[-\frac{t^{2}}{2 N}\right] d t
$$


Replace $-N$ by $-\infty$ as lower limit. That introduces errors $O(\exp -N)$. The term that contains odd power of $t$ goes out:

$$
=\exp [N \log N-N] \int_{-\infty}^{\infty} \exp \left[-\frac{t^{2}}{2 N}\right] d t=\sqrt{2 \pi N} \exp [N \log N-N] .
$$

The next term in the exponential series, $O\left(t^{4} / N^{3}\right)$ contributes an additional fraction $O(1 / N)$ of this final result.

b)

\begin{tabular}{lccc}
\hline$z$ & Stirling & Exact & Error \% \\
\hline 3 & 1.92 & 2 & 4 \\
3.5 & 3.21 & 3.32 & 3.3 \\
10 & 359537 & 362880 & 0.9 \\
10.5 & 1123380 & 1133280 & 0.8 \\
\hline
\end{tabular}

Problem 1.7. A gambler $G$ is involved in a game that pays off $(100 \pm x) \%$ of his bet, with $x$ a fixed number in the range $100>x>0$. The sign \pm is perfectly random. He plays it $N$ times against a much richer opponent (the bank) $B$, starting with an initial stake $\$ D$. At each step he tenders his entire stake (including any winnings or losses.) Be warned, this is an example where the "most probable" outcome differs considerably from the average.

(A) Show that for any $x$ and despite any fluctuations, with greatest probability $G$ 's stake must ultimately go to zero exponentially when $N>10^{4}$ is large. Is this paradoxical?

(B) Show that if, on the other hand, all winning (and losing) streaks of arbitrary lengths are allowed. $G$ ends up with precisely his initial stake $\$ D$ on average.

(C) By how small an amount must the odds change in order that, on average, $G$ always wins (or loses) consistently at large $N$ ? 
Solution: When calculating the most probable, we find:

$$
\begin{aligned}
\$ D\left(1+\frac{x}{100}\right)^{n_{1}}\left(1-\frac{x}{100}\right)^{n_{2}} & =\$ D\left(1-\frac{x^{2}}{10^{4}}\right)^{N / 2}\left(\frac{1+\frac{x}{100}}{1-\frac{x}{100}}\right)^{\Delta n} \\
& =\$ D e^{-\frac{N}{2} \log \left(\frac{1}{1-\frac{x^{2}}{10^{4}}}\right)}
\end{aligned}
$$

ignoring $\Delta n$ which vanishes on average and in any event, scales as $N^{1 / 2} \ll N$. This result should discourage any potential gambler.

However, when calculating the gambler's average net worth after $N$ plays, one has to include all the improbable runs, some with exponentially large winnings and losses. The exact formula for the average shows no change after $N$ plays:

$$
\begin{aligned}
\$ D\left\langle\left(1+\frac{x}{100}\right)^{n_{1}}\left(1-\frac{x}{100}\right)^{n_{2}}\right\rangle & \\
& =\$ D \sum_{n=0}^{N}\left(\begin{array}{c}
N \\
n
\end{array}\right)\left(1+\frac{x}{100}\right)^{n}\left(1-\frac{x}{100}\right)^{N-n} 2^{-N} \\
& \equiv \$ D\left(1+\frac{x}{200}-\frac{x}{200}\right)^{N}=\$ D
\end{aligned}
$$

i.e., no losses or gains, regardless of $N$. Of course, if the odds were stacked - even modestly — to the opponent, say $(1+x / 100)$ for each win and $(1-y / 100)$ for each loss, with $y=x+\varepsilon$, then even the average decreases exponentially as the reader easily verifies.

Problem 2.1. Show that the equilibrium magnetization in this model is $\langle M\rangle=N m_{0} \tanh \beta e_{0}$.

\section{Solution:}

$$
\begin{aligned}
\langle M\rangle & =\left\langle N m_{0}(2 x-1)\right\rangle=N m_{0}(2 \tilde{x}-1) \\
& =N m_{0}\left(\frac{2}{1+e^{-2 \beta \varepsilon_{0}}}-1\right)=N m_{0}\left(\frac{1-e^{-2 \beta \varepsilon_{0}}}{1+e^{-2 \beta \varepsilon_{0}}}\right) \\
& =N m_{0} \times \frac{e^{\beta \varepsilon_{0}}-e^{-\beta \varepsilon_{0}}}{e^{\beta \varepsilon_{0}}+e^{-\beta \varepsilon_{0}}}=N m_{0} \tanh \left(\beta \varepsilon_{0}\right) .
\end{aligned}
$$


Problem 2.2. (A) Show that the variance in the number of spins up is $\left\langle\left(n_{1}-\tilde{n}_{1}\right)^{2}\right\rangle / N=\frac{1}{4 \cosh ^{2} \beta e_{0}}$ and thus: $\left\langle\left(M\left(n_{1}\right)-\langle M\rangle\right)^{2}\right\rangle$ is extensive. (B) Is $\left\langle\left(F\left(n_{1}\right)-F\right)^{2}\right\rangle$ also extensive? Calculate this using $Q_{N}(x)$ and discuss.

Solution: (B) We know $F(x)-F(\tilde{x})=A N(x-\tilde{x})^{2}$ where $A>0$. Then,

$$
\begin{aligned}
\left\langle(F(x)-F(\tilde{x}))^{2}\right\rangle & =\frac{\int d x\left(A N x^{2}\right)^{2} e^{-\beta A N x^{2}}}{\int d x e^{-\beta A N x^{2}}} \\
& =\frac{1}{2 \beta^{2}},
\end{aligned}
$$

independent of the parameter $A N$, after shifting the origins of the integrations. Thus the rms "noise" in $F$ which was expected to be smaller than $O(N)$ is not even $O\left(N^{1 / 2}\right)$; it is actually $O\left(N^{0}\right)$ - independent of the size of the system, and vanishes as $T \rightarrow 0$. So, unlike all other thermodynamic functions the free energy is truly "stationary."

Problem 2.5. Calculate and plot the internal energy per site $-J s^{2}\langle\cos \theta\rangle$ and its derivative w.r. to $T$, the specific heat, for the classical Heisenberg model of $\S 2.9$, and compare with Fig. 2.2. Calculate and plot the entropy $\mathscr{S}=-\partial F / \partial T$ with the help of the expressions in Eqs. (2.15) and (2.16) and compare with the curve shown in Fig. 2.3. At what temperature does $\mathscr{S}$ vanish and change sign? 
Solution: This problem illustrates a number of concepts, so we shall dwell on it. Given the solid angle $d \Omega=d \varphi d \theta \sin \theta$ where $\theta$ is the angle between two neighbor spins and the Boltzmann weight factor for each angle, $\exp \left(\frac{J s^{2}}{k T} \cos \theta\right)$,

$$
\begin{aligned}
\langle\cos \theta\rangle_{T A} & =\int d \Omega \cos \theta \exp \left(\frac{J s^{2}}{k T} \cos \theta\right) \div \int d \Omega \exp \left(\frac{J s^{2}}{k T} \cos \theta\right) \\
& =\int d(\cos \theta) \cos \theta \exp \left(\frac{J s^{2}}{k T} \cos \theta\right) \div \int d(\cos \theta) \exp \left(\frac{J s^{2}}{k T} \cos \theta\right) \\
& =\int_{-1}^{1} d u u \exp (A u) \div \int_{-1}^{1} d u \exp (A u) \quad\left(\text { with } A \equiv \frac{J s^{2}}{k T}\right) \\
& =\frac{d}{d A} \log \int_{-1}^{1} d u \exp (A u)=\frac{d}{d A} \log \left(\frac{e^{A}-e^{-A}}{A}\right) \\
& =\operatorname{coth} A-\frac{1}{A}=\operatorname{coth} \frac{J s^{2}}{k T}-\frac{k T}{J s^{2}} .
\end{aligned}
$$

The energy per bond is thus, $E /(N-1)=-J s^{2} \operatorname{coth} \frac{J s^{2}}{k T}+k T$.

Omitting end effects, the free energy for a classical Heisenberg model chain is:

$$
\begin{aligned}
F & =-(N-1) k T \log \left\{4 \pi\left(k T / J s^{2}\right) \sinh \left(J s^{2} / k T\right)\right\} \\
& =-(N-1) k T[\log (4 \pi / A)+\log \sinh A] .
\end{aligned}
$$

The entropy is:

$$
\begin{aligned}
\mathscr{S}_{\text {Heis }} /(N-1) & =k \frac{E /(N-1)-F /(N-1)}{k T} \\
& =k(-A \operatorname{coth} A+1+\log (4 \pi / A)+\log \sinh A) .
\end{aligned}
$$

This vanishes at $A=17.0795$.

The specific heat: $c=C /(N-1)$, i.e. $c_{\text {Heis }}=T(d A / d T)\left(\partial \mathscr{S}_{\text {Heis }} / \partial A\right)=$ $k\left\{1-(A / \sinh A)^{2}\right\}$. This formula coincides with the "classical" formula for 2 rotational degrees of freedom per bond at low $T$. The classical way of counting degrees of freedom and assigning $k / 2$ per degree of freedom fails here at high $T$ where the specific heat actually vanishes. 
In the figures we compare these results with those for a similar Ising model chain, e.g., $c_{I}=k(A / \cosh A)^{2}$.

Problem 3.2. An "electron gas" (i.e. metal) has low-temperature heat capacity $c(T)=\gamma T \rightarrow 0$ as $T \rightarrow 0$. The low-temperature heat capacity of acoustic phonons is $c(T) \propto T^{3}$, also vanishing in the $\lim \cdot T \rightarrow 0$. Show both examples are compatible with the Third Law. By way of contrast, consider an hypothetical system $A$, in which $c_{A}(T)=c_{0} / \log \left(1+T_{0} / T\right)$, with $c_{0}$ and $T_{0}$ fixed parameters. At $T=0, c_{A}(T)$ also vanishes. Nevertheless, $A$ violates the Third Law as given above. Show this.

\section{Solution:}

$$
\begin{gathered}
c(T)=\gamma T \Rightarrow \mathscr{S}(T)=\int_{0}^{T} d T^{\prime} \frac{c\left(T^{\prime}\right)}{T^{\prime}}=\int_{0}^{T} d T^{\prime} \frac{\gamma T^{\prime}}{T^{\prime}}=\gamma T=0 \quad \text { as } T \rightarrow 0, \\
c(T) \propto T^{3} \Rightarrow S(T)=\int_{0}^{T} d T^{\prime} \frac{c\left(T^{\prime}\right)}{T^{\prime}}=\int_{0}^{T} d T^{\prime} \frac{\gamma T^{\prime 3}}{T^{\prime}}=\gamma \frac{T^{3}}{3}=0 \quad \text { as } T \rightarrow 0 .
\end{gathered}
$$

System $A$. In the integration, let $y=\log \frac{T_{o}}{T}$, then:

$$
\mathscr{S}(T)=\int_{0}^{T} \frac{c_{o} / T}{\log \frac{T_{o}}{T}+\log \left(1+\frac{T}{T_{o}}\right)} d T=c_{o} \int_{\log \frac{T_{0}}{T}}^{\infty} \frac{d y}{y+\log \left(1+e^{-y}\right)} .
$$

Integrand fails to vanish sufficiently fast at $y \rightarrow \infty$ (equivalent to $T \rightarrow 0$ ) so integral is $\infty$. 
Problem 4.1. The joint partition function of two separate, noninteracting species is the product of the two: $Z=Z\left(N_{A}, V_{A}, T_{A}\right) \times$ $Z\left(N_{B}, V_{B}, T_{B}\right)$. Calculate the total free energy when $T_{A}=T_{B}=T$. Calculate the "entropy of mixing" and the change in total energy after the two species are merged, using the total free energy derived from $Z\left(N_{A}, V_{A}+V_{B}, T\right) \times Z\left(N_{B}, V_{A}+V_{B}, T\right)$. What if the two species are indistinguishable? Discuss this in the context of the Second Law and the energy required in gaseous isotope separation.

\section{Solution:}

$$
\begin{aligned}
& Z_{A}\left(N_{A}, V_{A}, T\right)=\frac{V_{A}^{N_{A}}}{N_{A} !}\left(\frac{\sqrt{2 \pi m K T}}{h}\right)^{3 N_{A}}=\frac{V_{A}^{N_{A}}}{N_{A} !} G(T)^{3 N_{A}}=e^{-\beta F_{A}}, \\
& Z_{B}\left(N_{B}, V_{B}, T\right)=\frac{V_{B}^{N_{B}}}{N_{B} !}\left(\frac{\sqrt{2 \pi m K T}}{h}\right)^{3 N_{B}}=\frac{V_{B}^{N_{B}}}{N_{B} !} G(T)^{3 N_{B}}=e^{-\beta F_{B}} . \\
& \mathscr{S}_{A}=k \ln Z_{A}+\frac{3}{2} k N_{A}=k\left[N_{A}\left(\log V_{A}+3 \log G(T)+\frac{3}{2}\right)-\log N_{A} !\right], \\
& \mathscr{S}_{B}=k \ln Z_{B}+\frac{3}{2} k N_{B} .
\end{aligned}
$$

Before mixing: $N=N_{A}+N_{B}, Z_{\text {separate }}=Z_{A} Z_{B}$ (independent containers), hence

$$
\begin{aligned}
& F_{\text {separate }}=F_{A}+F_{B}, \quad \mathscr{S}_{\text {separate }}=\mathscr{S}_{A}+\mathscr{S}_{B} \quad \text { and } \\
& E_{\text {separate }}=\frac{\partial(\beta F)}{\partial \beta}=k T^{2} \frac{\partial\left(\log Z_{A} Z_{B}\right)}{\partial T}=\left(N_{A}+N_{B}\right) \frac{3 k T}{2}=\frac{3 N k T}{2} .
\end{aligned}
$$

After mixing: (for distinguishable particles): $V=V_{A}+V_{B}$ and

$$
\begin{aligned}
Z_{\text {after }} & =Z\left(N_{A}, V, T\right) \times Z\left(N_{B}, V, T\right)=\frac{(V)^{N_{A}+N_{B}}}{N_{A} ! N_{B} !} G(T)^{3 N}, \\
\mathscr{S}_{\text {after }} & =k\left[\left(\left(N_{A}+N_{B}\right)\left(\ln V+3 \ln G(T)+\frac{3}{2}\right)-\log \left(N_{A} ! N_{B} !\right)\right]\right. \\
& =\mathscr{S}_{\text {before }}+\Delta \mathscr{S}
\end{aligned}
$$


where

$$
\Delta \mathscr{S}=k\left[N_{A} \log \frac{V_{A}+V_{B}}{V_{A}}+N_{B} \log \frac{V_{A}+V_{B}}{V_{B}}\right]
$$

or, (letting $x_{A}=N_{A} / N$, etc.),

$$
\begin{gathered}
\Delta \mathscr{S}=k N\left[x_{A} \log \left(1+V_{B} / V_{A}\right)+x_{B} \log \left(1+V_{A} / V_{B}\right)\right] \\
E_{\text {after }}=\frac{\partial(\beta F)}{\partial \beta}=k T^{2}\left(3 N_{A}+3 N_{B}\right) \frac{\partial \ln G(T)}{\partial T}=E_{\text {separate }}=\frac{3 N k T}{2} .
\end{gathered}
$$

Change in Total Energy $\Delta E=0$. Thus, entropy of mixing $\Delta \mathscr{S}$ is all that changes. If $V_{A}=V_{B}, \Delta \mathscr{S}=k N \log 2$.

For indistinguishable particles with same densities $\frac{N_{A}}{V_{A}}=\frac{N_{B}}{V_{B}}$ and T; after mixing, $Z_{\text {after }}=\frac{\left(V_{A}+V_{B}\right)^{N_{A}+N_{B}}}{\left(N_{A}+N_{B}\right) !} G(T)^{3 N}$. Using Stirling's approximation, we find $\log Z_{\text {after }}=\log Z_{\text {separate, }} \therefore \Delta F=\Delta E=0$.

Problem 4.3. The microcanonical partition function $W$ conserves energy and numbers of particles. Explicitly: $W=\operatorname{Tr}\{\delta(E-H)\}$. Using the nowfamiliar representation of the delta function and the method of steepest descents valid at large $N$ and $E$, show this $W \equiv \exp \left(\mathscr{S}(E) / k_{B}\right)$ at a point of "steepest descents" (maximum amplitude and stationary phase) on the imaginary axis, $i t=z$. Relate the "effective" temperature to $E$ at the point of steepest descents by using $\frac{\partial \mathscr{S}(E)}{\partial z} \frac{\partial z}{\partial E}=\frac{\partial \mathscr{S}(E)}{\partial E}$ and invoking the definition of $T$ in Eqs. (3.5) and (3.10). Now that $E$ is "sharp", $T$ is not. What is the distribution of $T$ about its most probable value? of $\beta$ ?

\section{Solution:}

$$
\begin{aligned}
W=\operatorname{Tr}\left\{\frac{1}{2 \pi} \int e^{i t E-i t H} d t\right\} & =\frac{1}{2 \pi} \int e^{i t E} \operatorname{Tr}\left\{e^{-i t H}\right\} d t \\
& =\frac{1}{2 \pi} \int e^{i t E} e^{\Phi(t)} d t .
\end{aligned}
$$


From Thermodynamics: $E=\frac{\partial(\beta F)}{\partial \beta}$.

In our integral, by steepest descents: $E+\frac{\partial \Phi(t)}{\partial(i t)}=0$ or $E=-\frac{\partial \Phi(t)}{\partial(i t)}$.

$\therefore$ We identify: $\beta=i t$ and $\Phi=-\beta F$ at this point. Then shifting or relocating the origin of the integration to that same point,

$$
W=e^{\beta(E-F)} \frac{1}{2 \pi} \int d z e^{-\frac{1}{2} z^{2} \Gamma}=e^{S / k} \frac{1}{2 \pi} \int d z e^{-\frac{1}{2} z^{2} \Gamma}
$$

where

$$
\Gamma=-\frac{d^{2}(\beta F)}{d \beta^{2}}>0 .
$$

The integral factor does not scale exponentially with $V$ or $N$ but merely as $1 / \sqrt{\text { Vol. }}$ Therefore, to leading order in the size of the system, $k \log W=\mathscr{S}$. Even though $E$ is fixed,

$$
\left\langle(\beta-\bar{\beta})^{2}\right\rangle=\int d z z^{2} e^{-\frac{z^{2}}{2} \Gamma} / \int d z e^{-\frac{z^{2}}{2} \Gamma}=-2 \frac{\partial}{\partial \Gamma} \log \int e^{-\frac{z^{2}}{2} \Gamma}=\frac{1}{\Gamma} ;
$$

i.e., $T$ fluctuates! (But not in large systems for which $N \rightarrow \infty$ because $\Gamma \propto N$.

Problem 5.1. Prove the commutation relations in (5.5), using the definitions (5.1)-(5.3) for the various operators. Prove, then use the following identities to simplify the calculations:

1. $[a, A B]=A[a, B]+[a, A] B$, where $a, A$ and $B$ are arbitrary,

2. $[A, B]=-[B, A]$, and

3. $(A B)^{\dagger}=B^{\dagger} A^{\dagger}$

$A^{\dagger}$ is denoted "Hermitean conjugate" of $A$ if each is the transpose and the complex-conjugate of the other. The operators $a$ and $a^{+}$are Hermitean conjugates of one another, whereas $H$ is its own Hermitean conjugate i.e. it is "self-adjoint".

After inverting Eq. (5.2) for $p$ and $x$, expressing them as linear combinations of $a$ and $a^{+}$,

4. prove $x$ and $p$ in this representation are both self-adjoint. 


\section{Solution:}

1. $[a, A B]=a A B-A B a=(a A B-A a B)+(A a B-A B a)=[a, A] B+A[a, B]$

2. $[A, B]=A B-B A=-(B A-A B)=-[B, A]$

3. $(A B)^{\dagger}=(A B)^{T^{*}}=\left(B^{T} A^{T}\right)^{*}=B^{\dagger} A^{\dagger}$

$$
\begin{aligned}
& p=\left(\frac{h}{4 \pi} \sqrt{K M}\right)^{1 / 2}\left(a+a^{+}\right), \\
& x=i\left(\frac{h}{4 \pi \sqrt{K M}}\right)^{1 / 2}\left(a-a^{+}\right) .
\end{aligned}
$$

4. $p^{\dagger} \propto\left(a+a^{+}\right)^{+}=a^{+}+a \propto p$, therefore $p$ is self-adjoint $x^{\dagger} \propto i^{*}\left(a-a^{+}\right)^{+}=-i\left(a^{+}-a\right)=i\left(a-a^{+}\right) \propto x$, thus $x$ is also self-adjoint. Then, use (5.1) to prove the first identity in Eq. (5.5): $p x f(x)-x p f(x)=$ $(\hbar / i) f(x)$. Because this holds for all $f(x)$ it must be an operator identity. Use the representation of $p$ and $x$ in (3) above to show $\left[a, a^{+}\right]=1$ is also required for this operator identity to be true.

The other two commutator relations in (5.5) are special cases of (1) above.

Problem 5.4. Generalizing Eqs. (5.32) and (5.33), find $k T_{c}$ and the correct form of $M\left(T / T_{c}\right)$ in $3 \mathrm{D}, 4 \mathrm{D}, \ldots, \mathrm{dD}, \ldots$, as a function of $J S$, for $S=1 / 2,1, \ldots, \infty$.

Solution: Let $D=J S a_{0}^{2}, \varepsilon=D k^{2}, k=\sqrt{\frac{\varepsilon}{D}}, d \varepsilon=2 D k d k$. Then, use Problem 1.2 for $A_{d}$ to get:

$$
\left(\frac{L}{2 \pi}\right)^{d} \int d^{d} k \Phi\left(D k^{2}\right)=\frac{L^{d} A_{d}}{(2 \pi)^{d}} \int d k k^{d-1} \Phi\left(D k^{2}\right) \equiv L^{d} \int d \varepsilon \rho(\varepsilon) \Phi(\varepsilon) .
$$

With $\Phi=\frac{1}{e^{\beta \varepsilon}-1}$ and $\rho(\varepsilon)=$ const. $\times \varepsilon^{\frac{d}{2}-1}$, and integrating between limits 0 , $\infty$, obtain:

$$
\begin{aligned}
M & =N S\left(1-\frac{\text { const. } \int d \varepsilon \varepsilon^{\frac{d}{2}-1} \frac{1}{e^{\beta \varepsilon}-1}}{S}\right) \\
& =N S\left(1-\left(\frac{T}{T_{c}}\right)^{\frac{d}{2}}\right),
\end{aligned}
$$

which also serves to define $T_{c}$. 
Problem 5.6. Show that for free particles in $d$ dimensions, with $\varepsilon \propto$ $k^{2}, \rho(\varepsilon)=A_{d} \varepsilon^{-1+d / 2}$ for $\varepsilon>0$ and is $\equiv 0$ for $\varepsilon<0$. Determine $A_{d}$ for $d=1,2,3$.

Solution: Refer back to Problem 5.4, where we used $\rho(\varepsilon)=\frac{A_{d}}{(2 \pi)^{d}} k^{d-1} d k / d \varepsilon$, where $A_{d}$ is the area of the unit sphere in $d$ dimensions (cf. Problem 1.2). Then, evaluate both $d k / d \varepsilon$ and $k^{d-1}$ as functions of $\varepsilon$.

Problem 6.2. Typically one plots $c_{v} / T$ versus $T^{2}$ to obtain the coefficients of the linear term (the intercept) and of the cubic term, the slope. (A) Evaluate all the corrections $O\left(T / T_{F}\right)^{3}$ in the expression (6.20) and (B) compare them, in magnitude, to the low-temperature $T^{3}$ Debye law derived in Sec. 5.6, assuming exactly one electron per cell (i.e. $N=N_{0}=$ number of electrons $=$ number of atomic cells). Identify the important parameters and determine whether it is possible the electronic $T^{3}$ contributions might obscure those of the phonons in an ordinary metal at room temperature. Use the parameters: $T_{F}=100,000 \mathrm{~K}, \theta_{D}=200 \mathrm{~K}$ and room temperature $=$ $300 \mathrm{~K}$.

Solution: The $T^{3}$ contributions can be easily sorted out in the case of normal metals, where $T_{F}$ is several orders of magnitude greater than $T_{\theta}$. In that case, any $T^{3}$ contribution can be unambiguously attributed to lattice vibrations.

Problem 7.1. Using the laws of conservation of energy and momentum to somewhat "sharpen" the conclusions of Problem 7.1, prove that the 5 parameters in the asymptotic distribution function found above must be constants in time - that $\mathbf{a}, b, c$, cannot be periodic or aperiodic functions of $t$.

Solution: Calculate $d E(t) / d t=\int d^{3} p \varepsilon(p) \partial f / \partial t$ using Eq. (7.5).

Similarly for $\mathbf{P}(t)$. 
Problem 8.4. (a) Show that $e^{B \sigma_{j}}=\cosh B+\sigma_{j} \sinh B$ for $j=x, y$, or $z$. Using this, derive the expressions for $\Gamma$ in terms of $A$ and $\tilde{K}$ as given above, assuming $K$ is positive. Then, given (8.18b), prove the so-called "duality relations" (b) $\tanh K=e^{-2 \tilde{K}}$ and (c) $\sinh 2 \tilde{K} \sinh 2 K=1$.

Solution: There are several ways to prove part (a). The easiest is to write the identity, $\exp \gamma \equiv \cosh \gamma+\sinh \gamma$, then expand $\cosh$ in even powers of $\gamma$ (an even power of any of the three Pauli matrices $=$ unit matrix) and likewise for $\sinh \gamma$ (an odd power of any of the Pauli matrices $=$ itself). So if $\gamma=A \sigma$ where $\sigma$ is any of the Pauli matrices, $\cosh \gamma=\cosh A$, and $\sinh \gamma=\sigma \sinh A$. The remainder of the problem is treated similarly.

Problem 8.7. Prove $e^{i \pi c^{+} c} c^{+}=-c^{+}$and $e^{i \pi c^{+} c} c=+c$.

Solution: There are two possible states: $|0\rangle$ and $c^{+}|0\rangle$ (which can also be written $|n\rangle$ where $n=0$ or 1.) The operator $c^{+}$acting on the first of these yields the second, and acting on the second yields zero. Thus we only have to worry about the first. We calculate: $\exp \left(i \pi c^{+} c\right)|1\rangle$ which is by inspection $\exp (i \pi)|1\rangle=-c^{+}|0\rangle$, since $c^{+} c$ "reads" the number in $|n\rangle$.

Problem 9.5. Derive Eqs. (9.24) and (9.28) by expanding $\langle E\rangle^{2}$ as a sum of contributions from individual configurations.

Solution: Consider a sequence of configurations that generate a very large number of values of the energy, $\{E(t) \mid t=1, \ldots, N\}$. Consider the average of these values.

$$
\bar{E} \equiv \frac{1}{N_{M C}} \sum_{t} E(t)
$$

The error in that average is found from its variance. The square root of the variance gives the standard deviation, which is a measure of the error. When the thermal average is estimated from the width of a finite sampling, the usual factor of $\sqrt{N_{M C} /\left(N_{M C}-1\right)}$ must be included for an unbiased estimate of the error. 


$$
\begin{aligned}
\left\langle\bar{E}^{2}\right\rangle-\langle\bar{E}\rangle^{2} & =\left\langle\bar{E}^{2}\right\rangle-\langle E\rangle^{2} \\
& =\left\langle\frac{1}{N_{M C}} \sum_{t^{\prime}} E\left(t^{\prime}\right) \frac{1}{N_{M C}} \sum_{t} E\left(t^{\prime}+t\right)\right\rangle-\langle E\rangle^{2} \\
& =\left\langle\frac{1}{N_{M C}} \sum_{t^{\prime}}\left[E\left(t^{\prime}\right) E\left(t^{\prime}+t\right)-\langle E\rangle^{2}\right]\right\rangle \\
& =\frac{1}{N_{M C}} \sum_{t^{\prime}}\left[\left\langle E\left(t^{\prime}\right) E\left(t^{\prime}+t\right)\right\rangle-\langle E\rangle^{2}\right]
\end{aligned}
$$

where we have used the fact that $\left\langle E\left(t^{\prime}\right) E\left(t^{\prime}+t\right)\right\rangle$ is independent of $t^{\prime}$ in equilibrium.

Using the time-dependent correlation function,

$$
f_{E}(t) \equiv \frac{\left\langle E\left(t^{\prime}\right) E\left(t^{\prime}+t\right)\right\rangle-\langle E\rangle^{2}}{\left\langle E^{2}\right\rangle-\langle E\rangle^{2}}
$$

we can write the time-dependent correlations as

$$
\left\langle E\left(t^{\prime}\right) E\left(t^{\prime}+t\right)\right\rangle-\langle E\rangle^{2}=f_{E}(t)\left(\left\langle E^{2}\right\rangle-\langle E\rangle^{2}\right) .
$$

This gives us

$$
\left\langle\bar{E}^{2}\right\rangle-\langle\bar{E}\rangle^{2}=\frac{1}{N_{M C}} \sum_{t} f_{E}(t)\left(\left\langle E^{2}\right\rangle-\langle E\rangle^{2}\right) .
$$

Note that from the definition of the time-dependent correlation function, $f_{E}(t)=f_{E}(-t)$, so that we can write

$$
\left\langle\bar{E}^{2}\right\rangle-\langle\bar{E}\rangle^{2}=\frac{1}{N_{M C}} g_{E}=\frac{1}{N_{M C}}\left(1+2 \tau_{E}\right)
$$

where

$$
\tau_{E}=\sum_{t \geq 1} f_{E}(t)\left(\left\langle E^{2}\right\rangle-\langle E\rangle^{2}\right)
$$

Combining these equations, we find that the error is given by Eq. (9.24)

$$
\delta E=\sqrt{\left(\frac{g_{E}}{N_{M C}-1}\right)\left\langle(E-\langle E\rangle)^{2}\right\rangle} .
$$


Problem 10.2. Use Eq. (10.22) to derive Eq. (10.23) and a simple relationship between $K^{(1)}$ and $K_{o}^{(1)}$. Prove the inequality $K^{(1)}<K^{(0)}$ for any finite positive value of $K^{(0)}$.

\section{Solution:}

$$
\begin{aligned}
\exp \left[K^{(1)} s_{k}^{(1)} s_{k+1}^{(1)}+K_{o}^{(1)}\right]= & \exp \left[K^{(0)}\left(s_{2 k-1}^{(0)}+s_{2 k+1}^{(0)}\right)\right] \\
& +\exp \left[-K^{(0)}\left(s_{2 k-1}^{(0)}+s_{2 k+1}^{(0)}\right)\right] .
\end{aligned}
$$

Evaluate this equation for $s_{k}^{(1)} s_{k+1}^{(1)}=1$

$$
\exp \left[K^{(1)}+K_{o}^{(1)}\right]=\exp \left[2 K^{(0)}\right]+\exp \left[-2 K^{(0)}\right]
$$

and $s_{k}^{(1)} s_{k+1}^{(1)}=-1$

$$
\exp \left[-K^{(1)}+K_{o}^{(1)}\right]=2 .
$$

Dividing the first by the second, we find

$$
\exp \left[2 K^{(1)}\right]=\frac{1}{2}\left(\exp \left[2 K^{(0)}\right]+\exp \left[-2 K^{(0)}\right]\right)=\cosh \left(2 K^{(0)}\right) .
$$

Taking the logarithm of both sides, we find

$$
K^{(1)}=\frac{1}{2} \ln \cosh \left(2 K^{(0)}\right) .
$$

To prove that $K^{(1)}<K^{(0)}$, write this equation as

$$
\begin{aligned}
K^{(1)} & =\frac{1}{2} \ln \left[\frac{1}{2}\left(\exp \left[2 K^{(0)}\right]+\exp \left[-2 K^{(0)}\right]\right)\right] \\
& =\frac{1}{2} \ln \left[\frac{1}{2} \exp \left[2 K^{(0)}\right]\left(1+\exp \left[-4 K^{(0)}\right]\right)\right] \\
& =\frac{1}{2} \ln \left[\frac{1}{2}\right]+\frac{1}{2} \ln \left[\exp \left[2 K^{(0)}\right]\right]+\frac{1}{2} \ln \left[1+\exp \left[-4 K^{(0)}\right]\right] \\
& =K^{(0)}+\frac{1}{2} \ln \left[\frac{1+\exp \left[-4 K^{(0)}\right]}{2}\right]<K^{(0)} .
\end{aligned}
$$

The final inequality is due to the fact that the argument of the logarithm is less than one for any $K^{(0)}>0$. 
Problem 10.3. Calculate the free energy per site of the one-dimensional Ising model numerically by adding up the contributions to the renormalized Hamiltonians at each step. This calculation can be done using any programming language, or simply a spreadsheet. Your answer can be compared to the exact value previously derived in Eq. (2.11). How many numerical steps are required for an accuracy of 1 part in $10^{6}$ ?

Solution: This calculation can be very easily done with a spreadsheet program.

The answer to the last question is 20 steps. This can actually be seen without the computer program. The decimation transformation eliminates half the spins each time. Since the free energy is a finite quantity, the asymptotic contribution of each decimation should be half that of the previous step. The relative error should be roughly $2^{-n}$. Setting this equal to $10^{-6}$, we find $n=\log _{2} 10^{6}=19.93$. [Or you could just remember that $2^{10}=1024$.]

Problem 10.4. Derive Eq. (10.30) from the previous equations in this section.

The relevant equations are:

$$
\begin{aligned}
\xi^{(n+1)} & =\xi^{(n)} / b, \\
t & \sim\left(T_{c}-T\right), \\
t^{(n+1)} & =b^{y_{T}} t^{(n)},
\end{aligned}
$$

and

$$
\xi(t) \sim|t|^{-\nu}
$$

Equation (10.29) implies both

$$
\xi\left(t^{(n)}\right) \sim\left|t^{(n)}\right|^{-\nu}
$$

and

$$
\xi\left(t^{(n+1)}\right) \sim\left|t^{(n+1)}\right|^{-\nu} .
$$

Equation (10.1) then becomes

$$
\left|t^{(n+1)}\right|^{-\nu}=b^{-1}\left|t^{(n)}\right|^{-\nu}
$$


or

$$
\left|t^{(n+1)}\right|=b^{1 / \nu}\left|t^{(n)}\right|
$$

Comparison with Eq. (10.28) then gives

$$
\nu=\frac{1}{y_{T}} \text {. }
$$

Problem 10.5. Derive the last three relationships between the critical exponents and the eigenvalue exponents in Table 10.1.

\section{Solution:}

First Identity: Begin with the RG transformation of the free energy

$$
f(t, h)=g(t, h)+b^{-d} f\left(b^{y_{T}} t, b^{y_{h}} h\right) .
$$

To calculate the magnetization, $m$, we take the first derivative of $f$ with respect to the magnetic field. Ignoring constant factors, we have

$$
m(t, h)=\frac{\partial f(t, h)}{\partial h}=\frac{\partial g(t, h)}{\partial h}+b^{-d+y_{h}} m\left(b^{y_{T}} t, b^{y_{h}} h\right) .
$$

Evaluate this equation at zero magnetic field $(h=0)$ and choose $b$ such that

$$
b^{y_{T}} t=1 \text {. }
$$

Equation (10.2) then becomes

$$
m(t, 0)=\frac{\partial g(t, h)}{\partial h}+\left(t^{-1 / y_{T}}\right)^{-d+y_{h}} m(1,0) .
$$

But the critical exponent $\beta$ is conventionally designated to describe the leading singularity of the specific heat as a function of temperature. That is,

$$
m(t, 0)=A t^{\beta}+\cdots,
$$

where $A$ is a constant. Comparing with the previous equation and noting that $\frac{\partial g(t, h)}{\partial h}$ is presumed analytic, the leading non-analytic terms must be equal

$$
A t^{\beta}=t^{\left(d-y_{h}\right) y_{T}} m(1,0) .
$$

Comparing Eqs. (10.5) and (10.6) shows that the specific-heat exponent must be

$$
\beta=\left(d-y_{h}\right) / y_{T} .
$$


Second Identity: The second identity can be obtained by taking a second derivative of the free energy with respect to the magnetic field.

$$
\chi(t, h)=\frac{\partial^{2} f(t, h)}{\partial h^{2}}=\frac{\partial^{2} g(t, h)}{\partial h^{2}}+b^{-d+2 y_{h}} \chi\left(b^{y_{T}} t, b^{y_{h}} h\right)
$$

and again setting the magnetic field equal to zero and choosing $b$ such that $b^{y_{T}} t=1$.

$$
\chi(t, 0)=\frac{\partial^{2} f(t, 0)}{\partial h^{2}}=\frac{\partial^{2} g(t, 0)}{\partial h^{2}}+\left(h^{-1 / y_{h}}\right)^{-d+2 y_{h}} \chi(1,0) .
$$

Since the magnetic susceptibility diverges with an exponent $\gamma$

$$
\chi(t, 0)=A t^{-\gamma}+\cdots .
$$

Since $\frac{\partial^{2} g(t, 0)}{\partial h^{2}}$ should be analytic, we find

$$
A t^{-\gamma}=\frac{\partial^{2} g(t, 0)}{\partial h^{2}}+\left(h^{-1 / y_{h}}\right)^{-d+2 y_{h}} \chi(1,0)
$$

and

$$
\gamma=\left(2 y_{h}-d\right) / y_{T} .
$$

Third Identity: The last identity in the table can also be obtained from the equation

$$
m(t, h)=\frac{\partial f(t, h)}{\partial h}=\frac{\partial g(t, h)}{\partial h}+b^{-d+y_{h}} m\left(b^{y_{T}} t, b^{y_{h}} h\right)
$$

by setting $t=0$ and $b^{y_{h}} h=1$

$$
m(t, h)=\frac{\partial f(0, h)}{\partial h}=\frac{\partial g(0, h)}{\partial h}+\left(h^{-1 / y_{h}}\right)^{-d+y_{h}} m(0,1) .
$$

The exponent $\delta$ describes the power-law behavior of the magnetization as a function of magnetic field at the critical temperature

$$
\mathrm{m}=\mathrm{A}|h|^{1 / \delta}+\cdots .
$$

Since $\frac{\partial g(0, h)}{\partial h}$ should vanish by symmetry, we find

$$
|h|^{1 / \delta}=\left(|h|^{-1 / y_{h}}\right)^{-d+y_{h}}
$$

or

$$
\delta=y_{h} /\left(d-y_{h}\right)
$$


Problem 10.6. Derive the two identities: $\alpha+2 \beta+\gamma=2$ and $\gamma=\beta(\delta-1)$ from the relationships in Table 10.1.

Solution: From Table 10.1, we have

$$
\begin{aligned}
& \alpha=2-d / y_{T}, \\
& \beta=\left(d-y_{H}\right) / y_{T}
\end{aligned}
$$

and

$$
\gamma=\left(2 y_{H}-d\right) / y_{T}
$$

Forming the sum, we find:

$$
\begin{aligned}
\alpha+2 \beta+\gamma & =2-d / y_{T}+2\left(d-y_{h}\right) / y_{T}+\left(2 y_{h}-d\right) / y_{T} \\
& =2-d / y_{T}+2 d / y_{T}+2\left(-y_{h}\right) / y_{T}+\left(2 y_{h}\right) / y_{T}+(-d) / y_{T} \\
& =2 .
\end{aligned}
$$

For the second identity, use

$$
\delta=y_{h} /\left(d-y_{h}\right)
$$

and write $\beta(\delta-1)$ as

$$
\begin{aligned}
\beta(\delta-1) & =\left[\left(d-y_{h}\right) / y_{T}\right]\left(y_{h} /\left(d-y_{h}\right)-1\right) \\
& =\left(\frac{d-y_{h}}{y_{T}}\right)\left(\frac{y_{h}}{d-y_{h}}-\frac{d-y_{h}}{d-y_{h}}\right) \\
& =\left(\frac{d-y_{h}}{y_{T}}\right)\left(\frac{2 y_{h}-d}{d-y_{h}}\right) \\
& =\left(\frac{2 y_{h}-d}{y_{T}}\right)=\gamma .
\end{aligned}
$$

Problem 10.7. Derive Eqs. (10.42) and (10.43) from Eqs. (10.8), (10.14) and the definition of the partition function.

Solution: From the definition of the partition function for the $(n+1)$-level of RG iterations,

$$
Z^{(n+1)}=\sum_{\left\{s_{i}^{(n+1))}\right\}} \exp \left[\sum_{\alpha} K_{\alpha}^{(n+1)} S_{\alpha}^{(n+1)}\right],
$$


we can take a logarithmic derivative with respect to $K_{\gamma}^{(n+1)}$ to find

$$
\left\langle S_{\gamma}^{(n+1)}\right\rangle=\frac{1}{Z^{(n+1)}} \sum_{\left\{s_{i}^{(n+1))}\right\}} S_{\gamma}^{(n+1)} \exp \left[\sum_{\alpha} K_{\alpha}^{(n+1)} S_{\alpha}^{(n+1)}\right]
$$

taking a derivative of this expression with respect to $K_{\alpha}^{(n+1)}$ gives us

$$
\begin{aligned}
\frac{\partial\left\langle S_{\gamma}^{(n+1)}\right\rangle}{\partial K_{\alpha}^{(n+1)}=} & \frac{1}{Z^{(n+1)}} \sum_{\left\{s_{i}^{(n+1))}\right\}} S_{\gamma}^{(n+1)} S_{\alpha}^{(n+1)} \exp \left[\sum_{\alpha^{\prime}} K_{\alpha^{\prime}}^{(n+1)} S_{\alpha^{\prime}}^{(n+1)}\right] \\
& -\left(\frac{1}{Z^{(n+1)}} \sum_{\left\{s_{i}^{(n+1))}\right\}} S_{\gamma}^{(n+1)} \exp \left[\sum_{\alpha^{\prime}} K_{\alpha^{\prime}}^{(n+1)} S_{\alpha^{\prime}}^{(n+1)}\right]\right) \\
& \times\left(\frac{1}{Z^{(n+1)}} \sum_{\left\{s_{i}^{(n+1))}\right\}} S_{\alpha}^{(n+1)} \exp \left[\sum_{\alpha^{\prime}} K_{\alpha^{\prime}}^{(n+1)} S_{\alpha^{\prime}}^{(n+1)}\right]\right) \\
= & \left\langle S_{\gamma}^{(n+1)} S_{\alpha}^{(n+1)}\right\rangle-\left\langle S_{\gamma}^{(n+1)}\right\rangle\left\langle S_{\alpha}^{(n+1)}\right\rangle .
\end{aligned}
$$

To obtain the remaining identity, use Eq. (10.8)

$$
\exp \left[H^{(n+1)}\left(s^{(n+1)}\right)\right]=\sum_{\left\{s^{(n)}\right\}} T\left(s^{(n+1)}, s^{(n)}\right) \exp \left[H^{(n)}\left(s^{(n)}\right)\right]
$$

to write

$$
\begin{aligned}
Z^{(n+1)} & =\sum_{\left\{s_{i}^{(n+1))}\right\}} \exp \left[\sum_{\alpha} K_{\alpha}^{(n+1)} S_{\alpha}^{(n+1)}\right] \\
& =\sum_{\left\{s_{i}^{(n+1))}\right\}} \sum_{\left\{s^{(n)}\right\}} T\left(s^{(n+1)}, s^{(n)}\right) \exp \left[\sum_{\alpha} K_{\alpha}^{(n)} S_{\alpha}^{(n)}\right]
\end{aligned}
$$

and

$$
\left\langle S_{\gamma}^{(n+1)}\right\rangle=\frac{1}{Z^{(n+1)}} \sum_{\left\{s_{i}^{(n+1))}\right\}} S_{\gamma}^{(n+1)} \sum_{\left\{s^{(n)}\right\}} T\left(s^{(n+1)}, s^{(n)}\right) \exp \left[\sum_{\alpha} K_{\alpha}^{(n)} S_{\alpha}^{(n)}\right] .
$$


Now taking a derivative with respect to $K_{\beta}^{(n)}$, we find

$$
\begin{aligned}
\frac{\partial\left\langle S_{\gamma}^{(n+1)}\right\rangle}{\partial K_{\beta}^{(n)}}= & \frac{1}{Z^{(n+1)}} \sum_{\left\{s_{i}^{(n+1))}\right\}} S_{\gamma}^{(n+1)} \sum_{\left\{s^{(n)}\right\}} T\left(s^{(n+1)}, s^{(n)}\right) S_{\beta}^{(n)} \exp \left[\sum_{\alpha} K_{\alpha}^{(n)} S_{\alpha}^{(n)}\right] \\
& -\left(\frac{1}{Z^{(n+1)}} \sum_{\left\{s_{i}^{(n+1))}\right\}} S_{\gamma}^{(n+1)} \sum_{\left\{s^{(n)}\right\}} T\left(s^{(n+1)}, s^{(n)}\right) \exp \left[\sum_{\alpha} K_{\alpha}^{(n)} S_{\alpha}^{(n)}\right]\right) \\
& \times\left(\frac{1}{Z^{(n+1)}} \sum_{\left\{s_{i}^{(n+1))}\right\}} \sum_{\left\{s^{(n)}\right\}} T\left(s^{(n+1)}, s^{(n)}\right) S_{\beta}^{(n)} \exp \left[\sum_{\alpha} K_{\alpha}^{(n)} S_{\alpha}^{(n)}\right]\right)
\end{aligned}
$$

or

$$
\frac{\partial\left\langle S_{\gamma}^{(n+1)}\right\rangle}{\partial K_{\beta}^{(n)}}=\left\langle S_{\gamma}^{(n+1)} S_{\beta}^{(n)}\right\rangle-\left\langle S_{\gamma}^{(n+1)}\right\rangle\left\langle S_{\beta}^{(n)}\right\rangle .
$$




\section{Bibliography}

Allen, P. B., Phys. Rev. Lett. 59, 1460 (1987).

Amit, S. J., Field Theory, the Renormalization Group and Critical Phenomena (McGraw-Hill, New York, 1978).

Astumian, R. D. and P. Hänggi, in Physics Today, Nov. 2002, pp. 33-39.

Baake, E., M. Baake and H. Wagner, Phys. Rev. Lett. 78, 559 (1997); Nonequilibrium Energy Transport, T. Antal, Z. Racz and L. Sasvari, ibid., 167.

Bardeen, J. and J. R. Schrieffer, Recent Developments in Superconductivity, Vol. III, Chapter 6; C. J. Gorter, ed., Progr. Low Temp. Phys. (North-Holland, 1961). Bardeen, J., L. N. Cooper and J. R. Schrieffer, Phys. Rev. 106, 162 (1957), and 108, 1175 (1957).

Barker, J. A. and D. Henderson, Rev. Mod. Phys. 48, 587 (1976).

Berg, B. A. and T. Neuhaus, Phys. Rev. Lett. 68, 9 (1992).

Binder, K. and A. P. Young, Rev. Mod. Phys. 58, 801-976 (1986).

Binney, J. J., N. J. Dowrick, A. J. Fisher and M. E. J. Newman, The Theory of Critical Phenomena (Clarendon Press, Oxford, 1992).

Bogolubov, N. N., J. Phys. USSR 11, 23 (1947); P. Paul and B. Sutherland's "Interacting one-dimensional Bose gas: beyond Bogolubov," Phys. Rev. A 62, 055601 (2000).

Bortz, A. B., M. H. Kalos and J. L. Lebowitz, J. Comp. Phys. 17, 10 (1975).

Bouzida, D., S. Kumar and R. H. Swendsen, "Efficient Monte Carlo Methods for Computer Simulation of Biological Molecules," Phys. Rev. A 45, 8894-8901 (1992).

Brown, J. and A. Carrington, Rotational Spectroscopy of Diatomic Molecules (Cambridge Univ. Press, Cambridge, 2003).

Brush, S. G. in Rev. Mod. Phys. 39, 883 (1967); L. Onsager, Phys. Rev. 65, 117 (1944); B. Kaufman, Phys. Rev. 76, 1232 (1949); L. Onsager, Nuovo Cimento (Suppl.) 6, 261 (1949); C. N. Yang, Phys. Rev. 85, 808 (1952); T. Schultz, D. Mattis and E. Lieb in Rev. Mod. Phys. 36, 856 (1964).

Cápek, V. and D. P. Sheehan, Challenges to the Second Law of Thermodynamics, Theory and Experiment (Springer, Dodrecht (NL) and Norwell, MA, 2005). 
Cardy, J., Scaling and Renormalization in Statistical Physics (Cambridge Univ. Press, Cambridge, 1996), p. 28.

Chamberlin, R. V., On Non-Extensive Energies, Science 298, 1172 (2002).

deJongh, L. and A. Miedema, Adv. Phys. 23, 1-260 (1974).

Deutscher, G., New Superconductors: from Granular to High $T_{c}$ (World Scientific, Singapore, 2006), esp. Chapters 3 and 4 on the thermodynamics.

Doi, M., J. Phys. A 9, 1465 and 1479 (1976).

Dugdale, J. S., Entropy and Its Physical Meaning (Taylor \& Francis, London, 1996), p. 145 .

Evans, M., N. Hastings and B. Peacock, Statistical Distributions (Wiley, New York, 1993). This 2nd edition describes 39 "major" distributions.

Ferrenberg, A. M. and R. H. Swendsen, Phys. Rev. Lett. 61, 2635 (1988); ibid. 63, 1195 (1989).

Feynman, R. P., Phys. Rev. 91, 1291 and 1301 (1953); 94, 262 (1954) and R. P. Feynman and M. Cohen, Phys. Rev. 102, 1189 (1956).

Feynman, R. P., Rev. Mod. Phys. 29, 205-212 (1957).

Feynman, R. P., Statistical Mechanics (Addison-Wesley, Reading, 1998), p. 312.

Fisher, M. E., Rep. Progr. Phys. 30, 615 (1967).

Forgacs, G., Phys. Rev. B 22, 4473 (1980) and references therein.

Fuchs, K., Proc. Camb. Phi. Soc. 34, 100 (1938); E. H. Sondheimer, Adv. Phys. 1, 1 (1952); Proc. Roy. Soc. A 224, 260 (1954); F. S. Ham and D. C. Mattis, IBM Journal 4, 143 (1960).

Goodstein, D. H., States of Matter (Dover, New York, 1985).

Groeneveld, J., Phys. Lett. 3, 50 (1962); J. Stephenson, Phys. Chem. Liq. 10, 229 (1981); "Hard-and Soft-Core Equations of State", in Proc. 8th Symposium on Thermophysical Properties (ASM, New York), Vol. 1, J. Sengers, ed., [book \# 100151], pp. 38-44.

Guggenheim, E. A., J. Chem. Phys. 13, 253 (1945).

Gupta, R. and R. Cordery, Phys. Lett. A 105, 415 (1984).

Happel, H., Ann. Physik 21, 342 (1906); B. Nijboer and L. van Hove, Phys. Rev. 85, 777 (1952); F. H. Ree and W. G. Hoover, J. Chem. Phys. 40, 939 (1964). Hill, T. L., Nanothermodynamics, Nanoletts. 1, 111 (2001).

Hill, T. L., Thermodynamics of Small Systems (Dover reprints, NY, 1994).

Hoover, W. G., Phys. Rev. A 31, 1695 (1983).

Itzykson, C., H. Saleur and I.-B. Zuber, Conformal Invariance and Applications to Statistical Mechanics (World Scientific, Singapore, 1988).

Kadanoff, L. P., Physics 2, 263 (1967).

Kadanoff, L. P. et al., Rev. Mod. Phys. 39, 395-431 (1967).

Kiess, E., Am. J. Phys. 55, 1006 (1987).

Kosterlitz, J. and D. Thouless, J. Phys. C 6, 1181 (1973); V. L. Berezinskii, Sov. Phys. (JETP) 32, 493 (1971); D. Mattis, Phys. Lett. 104, 357 (1984). 
Kumar, S., D. Bouzida, R. H. Swendsen, P. A. Kollman and J. M. Rosenberg, J. Comp. Chem. 13, 1011 (1992); S. Kumar, J. M. Rosenberg, D. Bouzida, R. H. Swendsen and P. A. Kollman, J. Comp. Chem. 16, 1339 (1995).

Langenberg, D., D. Scalapino and B. Taylor, Scientific American 214, 30 (1966).

Lee, T. D., K. Huang and C. N. Yang, Phys. Rev. 106, 1135 (1957).

London, F., and H. London, Proc. Roy. Soc. (London) A 149, 71 (1935) and Physica 2, 341 (1935).

Ma, S.-K., Phys. Rev. Lett. 37, 461 (1976).

Mahan, G. D., Many-Particle Physics (Plenum, New York, 1990), 2nd Edition.

March, N. H. and M. P. Tosi, Introduction to Liquid State Physics (World Scientific, Singapore, 2002), p. 184.

Martin, P., Potts Models and Related Problems in Statistical Mechanics (World Scientific, Singapore, 1991).

Massalski, T. D., ed., Binary Alloy Phase Diagrams (ASM International, Materials Park, Ohio, 1990).

Mattis, D., The Many-Body Problem (World Scientific, 1994), Chap. 1.

Mattis, D. C. and E. H. Lieb, J. Math. Phys. 2, 602 (1961).

Mattis, D. C. and M. L. Glasser, Rev. Mod. Phys. 70, 979 (1998).

Mattis, D. C., The Theory of Magnetism Made Simple (World Scientific, Singapore, 2006).

Mattis, D. C., A. M. Szpilka and H. Chen, Mod. Phys. Lett. B 3, 215 (1989).

Mayer, J. and M. Mayer, Statistical Mechanics (J. Wiley, New York, 1940); H. L. Frisch and J. L. Lebowitz, The Equilibrium Theory of Classical Fluids (Benjamin, New York, 1964).

Mehta, M. L., Random Matrices (Academic, New York, 1967).

Mermin, N. D. and H. Wagner, Phys. Rev. Lett. 17, 1133 and 1307 (1966).

Molina, M. and D. Mattis, Phys. Lett. A 159, 337 (1991); J. Yáñez, M. Molina and D. Mattis, ibid. 288, 277 (2001); N. Xu et al., Phys. Rev. Lett. 98, 175502 (2007).

Müller-Krumbhaar, H. and K. Binder, J. Stat. Phys. 8, 1 (1973).

Newell, G. and E. Montroll, Rev. Mod. Phys. 25, 353 (1953).

Nienhuis, B. and M. Nauenberg, Phys. Rev. Lett. 35, 477 (1975).

Nishimori, H., Statistical Physics of Spin Glasses and Information Processing (Oxford Univ. Press, Oxford, 2001).

Nose, S., J. Chem. Phys. 81, 511 (1984).

Onsager, L., Phys. Rev. 37, 405; 38, 2265 (1931); L. Onsager and S. Machlup, Phys. Rev. 91, 1505, 1512 (1953).

Palmeri, J., J. Stat. Phys. 58, 885 (1990).

Paul, P. and B. Sutherland, Interacting Electron Gas: beyond Bogolubov, Phys. Rev. A 62, 055601 (2000).

Rayleigh, L. (J. W. Strutt), Phil. Mag. 26, 776 (1913).

Rickayzen, G., Theory of Superconductivity (Wiley, New York, 1965). 
Rosenberg, R., "Why is Ice Slippery?" Physics Today, pp. 50-55, December, 2005. Schrieffer, J. R., Theory of Superconductivity (Benjamin, New York, 1964).

Sheehan, D. P., ed., Quantum Limits to the Second Law (A.I.P., Melville, NY, 2002). Simon, F. , Ergeb. Exakt. Naturwiss. 9, 222 (1930).

Smith, A., J. Janak and R. Adler, Electronic Conduction in Solids (McGraw-Hill, New York, 1967).

Sollich, P. et al, J. Phys. Soc. Jpn. 69, 3200 (2000).

Stanley, H. E., Introduction to Phase Transitions and Critical Phenomena (Oxford Univ. Press, Oxford, 1971).

Svensson, E. C., Proc. of Los Alamos Workshop, LA-10227-C, Vol. 2, 1984, p. 456.

Swendsen, R. H., "Monte Carlo Renormalization," in Real-Space Renormalization; eds. Th. W. Burkhardt and J. M. J. van Leeuwen, Topics in Current Physics (Springer, Berlin, 1982), Vol. 30.

Swendsen, R. H. and J.-S. Wang, "Replica Monte Carlo Simulation of Spin Glasses," Phys. Rev. Lett. 57, 2607 (1986).

Swendsen, R. H., Am. J. Phys. 74, 187 (2006) and references therein.

Swendsen, R. H., Phys. Rev. B 30, 3866, 3875 (1984).

Swendsen, R. H., Phys. Rev. Lett. 42, 859 (1979).

Sykes, M. et al., J. Phys. A 5, 624ff, 640ff, 667ff (1972), A6, 1498, 1506 (1973).

Tata, B. V. R. et al., Phys. Rev. Lett. 84, 3626 (2000).

Toussaint, D. and F. Wilczek, J. Chem. Phys. 78, 2642 (1983).

Waldmann, L., Handbuch d. Physik, Vol. 12.

Wang, F. and D. P. Landau, Phys. Rev. Lett. 86, 2050 (2001); Phys. Rev. E 64, 056101 (2001).

Wannier, G., Phys. Rev. 79, 357 (1950); C. Domb, Adv. Phys. 9, 149-361 (1960).

Widom, B., J. Chem. Phys. 43, 3892, 3896 (1965).

Widom, B., J. Chem. Phys. 43, 3898 (1965).

Widom, B., Science 157, 375 (1967).

Wilson, K. G., Rev. Mod. Phys. 47, 773 (1975).

Wolff, U., Phys. Rev. Lett. 62, 361 (1989).

Wu, F. Y., Rev. Mod. Phys. 54, 235 (1982).

Yeomans, J. M., Statistical Mechanics of Phase Transitions (Clarendon Press, Oxford, 1992).

Ziman, J., Electrons and Phonons (Oxford Univ. Press, London, 1960), pp. 280 ff.

Zubarev, D. N., Sov. Phys. (Uspekhi) 3, 320-345 (1960) (English translation of the original in Usp. Fiz. Nauk 71, 71-116 (1960)). 


\section{Index}

addition theorem 3

adiabatic $27,41,44,54-56,99,100$

antiferromagnet $109,219,220,290$

approach to equilibrium 23, 41, 160, 161, 165, 166

area of unit sphere $7,306,317$

backscattering model 178

barometer equation $15,16,80,177$

BCS theory $59,125,140,149,152,156,158,211$

binomial $1-3,6,8-10,14,17,70$

Bogolubov inequality 290, 293

Bogolubov transformation 124, 125, 154, 157

Boltzmann equation $161,169,171-173$

Brillouin zone $88,106,108,211,280$

Brownian motion $5,10,57,280$

Carnot cycle $\quad 54-56$

Central Limit Theorem 10, 69

chain rule $37,38,44,272$

chemical potential $15,21,36,38,42,53,67,94,101,110,112,133-136,143$

coexistence curve 49,51

collision $10,13,20,160-165,167,168,171-178,180-182,185,187,189,193$

commutator algebra 96, 98, 154

completeness 164, 185, 186, 190

compressibility $26,37,43-46,48,50,51,53,115,227,242$

concavity theorem 63

conductivity $43,58,126,172,173,175-177,182-184,294$

configurational partition function $75,82,195,197,255$

Cooper pairs 149, 150, 158, 159 
correlation function $31,36,71-73,77,78,82,85,86,164,226,227,244,287,294$, 296-300, 319

Coulomb repulsion $143,144,146,147$

critical exponents $28,46,217-219,225-228,230,247,249-254,268,270-272$,

297,322

critical point $26,28,29,45-47,50-52,77,81,115,217,227,228,244,246,249$,

250, 254, 255, 260, 266-269, 271, 272, 292

Curie's law 26, 217, 224

cyclotron frequency 184

Debye integrals 107,109

decimation $263,265,321$

density matrix 62,200

density of states $($ dos $) \quad 102,108,135$

detailed balance 160, 163, 165, 168, 176, 236, 237, 241

diamagnetism 58

diatomic molecules $90-92,110$

diffusive motion $\quad 6,228$

Dirac delta function 11,13

disordered chain 32

Drude conductivity, 175

duality $19,38,206,214,230,318$

efficiency $56,233,237,238,241$

Einstein relation 176,177

elementary excitation $86,127-130,140,152$

energy gap $86,142,149,151,152,156-159,290$

ensemble $17,62,69,71,121,169,176,295$

enthalpy 39

entropy defined 69,99

equation of state $26,27,30,36,37,44,45,47,49,54,55,60,67,68,73,74,76$, $80,82,83,92,114,115,139,227$

ergodic $15,60,61,162,236,241$

exponent identities 271

Fermi function 134, 144, 146, 300

Fermi-Dirac gas $68,133,135,137,139,140,142,296$

Feynman's theorem 63

Fick's law 176

First Law $40,41,56,57$

fixed point $\quad 260,264-270,274,276,277$ 
Frobenius theorem 198, 203

frustration $\quad 219-222,224$

gamma function 6, 104

Gaussian potentials 204

generating function $4,14,107,170,283,285,286$

Gibbs' free energy $39,44,48,53$

Gibbs' phase rule 53

Green function 160, 279-281, 294, 297-301, 303, 304

ground state depletion 124

$H$-function 166

Hall constant 183

heat capacity $25,26,36,42,43,58,63,91,92,105,106,108,114,126,127,138$, 176,312

Heisenberg model $33-35,91,225,289,292,310,311$

Helmholtz free energy 38

high-temperature superconductors 159

ideal Bose condensation 113

in low dimensions 115

indistinguishable particles $\quad 69,132,314$

Ising ladder 207

Ising model 19, 20, 31-34, 86, 131, 132, 205, 207-209, 212, 217, 218, 222, 223, 227, 229, 231, 245-247, 249-253, 256, 258-260, 262, 263, 265-268, 274-277, 289, 312,321

Joule heating 43, 174, 175

junction 148, 174

lattice gas $19,20,30,86,112,228,229,289$

law of corresponding states $45,47,50,58,155,156$

Legendre transformation 39,70

Liouville operator 172

long-range order $27,28,31-33,50-52,84,109,126,216,279,288$

master equation 161,169

Maxwell construction $45,46,48,50,59$

Maxwell relations $23,37,40,44,272$

mean field (see molecular field) 26, 28-31, 46, 83, 166, 214, 217-219, 225, 228, 249, 254, 255, 287, 288, 295 
Mermin-Wagner theorem 279, 290

molecular dynamics $\quad 61,81,160,232$

molecular field (see mean field) 26

Morse potential 121

nanophysics 93

negative $T \quad 29$

Nernst $42,43,58,67,220$

normal distribution $9,10,12,16,244$

normal modes $88,96,105,123,125,160,204,211,279,300,304$

number representation $95,97,98$

Ohm's law 161,175

paramagnetism 21

partition function $62,65-71,75,82,91,92,105,120,133,135,145,195,197$, 205, 236, 255, 270, 273, 313, 314, 324

Pauli operators 207, 213

phase diagram $23,29,44,50,52-55,126,127,225,231$

phonons $42,82,90,94,105,106,108,127,128,139,142,149,157,172,173,180$, $181,279,288,289,312,317$

Planck's law 102, 165, 168, 169

Poisson distribution 8,171

Potts model 20, 229-231, 245, 289

quasiparticles $131,132,140,141,155-157$

radioactive decay $8,169 \mathrm{ff}, 171,286$

random matrix 16,302

random walk 5, 199, 235, 281, 303

relaxation-time approximation 182

renormalization $31,46,226,228,242,253-255,258-260,263,265,266,269,270$,

$272,275,278$

rotations $\quad 64,90-92,208,240,241$

scaling $\quad 46,226-228,231,247,251-253,255,270,276,277$

second derivatives $26,43,44,51,270,323$

Second Law 41, 45, 48, 56, 57, 66, 67, 161, 313

Slater determinant 132

sound waves $36,160,172,184,188,190,193,194$

spin glass $222,224,225,246$

spontaneous symmetry breaking $28,30,32,290,293$ 
Stirling's approximation $\quad 6-9,14,66,307,314$

superconductivity $43,57,132,140,149-152,156,158,159,212$

superfluid $\quad 68,78-80,110,115,126,127,228,295$

superfluidity $78,149,150,159$

susceptibility $25,26,28,43,53,63,135,137,141,217,218,224,225,227,242$,

$251,252,272,323$

temperature defined 234

Third Law 35, 42, 43, 139, 312

Toeplitz matrix 216, 303

Tonks' gas 82,118

trace $62,197,205,230$

transfer matrix $\quad 83,85,195,204-207,213,218,220,222,223,230$

transport $\quad 60,160,169,172,173,181,182,207,297$

triple point $52,53,77$

two-fluid model 149

van der Waals gas 44

variance $4,12,13,24,63,247,286,288,310,318$

virial $45,73,76,77,80,81$

zeta function $104,113,141$

Zipper 195-199, 203, 205, 207

Zipper ladder 198 